Sādhanā Vol. 28, Part 6, December 2003, pp. 975-990. ( ) Printed in India

\title{
Plane strain problem in microstretch elastic solid
}

\author{
RAJNEESH KUMAR ${ }^{1}$, RANJIT SINGH ${ }^{2}$ and T K CHADHA ${ }^{3}$ \\ ${ }^{1}$ Department of Mathematics, Kurukshetra University, Kurukshetra 136 119, India \\ ${ }^{2}$ Department of Mathematics, Govt. College, Patti 143 416, India \\ ${ }^{3}$ Department of Mathematics, Guru Nanak Dev University, Amritsar 143 005, India \\ e-mail: search@granth.kuk.ernet.in; rajneesh_kuk@rediffmail.com; \\ kalsi_ranjit@yahoo.com
}

MS received 17 February 2000; revised 1 August 2003

\begin{abstract}
The eigenvalue approach is developed for the two-dimensional plane strain problem in a microstretch elastic medium. Applying Laplace and Fourier transforms, an infinite space subjected to a concentrated force is studied. The integral transforms are inverted using a numerical technique to get displacement, force stress, couple stress and first moment, which are also shown graphically. The results of micropolar elasticity are deduced as a special case from the present formulation.
\end{abstract}

Keywords. Eigenvalue; microstretch elastic solid; two-dimensional plane problem; integral transform; impulsive force.

\section{Introduction}

It is well known that material response to external stimuli depends on the internal structure. Classical elasticity ignores this aspect by ascribing only translation degree of freedom of the material point of the body. The micropolar theory of Eringen (1966) includes the intrinsic rotation. Such a model can support body and surface couples and display the high frequency optical branch of the wave spectrum. Eringen (1971) extended his work to include the axial stretch during the rotation of molecules and developed the theory of micropolar elastic solid with stretch. Eringen (1990) also developed the theory of microstretch elastic solid in which he included microstructural expansions and contractions. Microstretch continuum is a model for Bravais lattice with basis on the atomic level, and two phase dipolar solids with a core on the macroscopic level. Composite materials reinforced with chopped elastic fibres, porous media whose pores are filled with gas or inviscid liquid, asphalt or other elastic inclusions and 'solid-liquid' crystals etc. are examples of microstretch solids.

Many researchers discussed various problems based on this theory (Kumar \& Gogna 1992; Iesan \& Nappa 1994; Iesan \& Quintanilla 1994; Iesan \& Pompei 1995; Bofilit \& Quintanilla 1995; Iesan \& Nappa 1995; Kumar \& Singh 1996; Singh \& Kumar 1998). The eigenvalue approach has been applied successfully by different researchers in different media including Das et al (1983), Mahalanabis \& Manna (1989), Saxena \& Dhaliwal (1990), Sharma \& Chand (1992), Sharma \& Kumar (1996). We have considered two-dimensional plane problem in microstretch elastic solid by employing the eigenvalue approach. 


\section{Basic equations}

The constitutive relations and field equations in microstretch solids are given by Eringen (1990)

$$
\begin{aligned}
& t_{i j}=\left(\lambda_{0} \phi^{*}+\lambda u_{r, r}\right) \delta_{i j}+\mu\left(u_{i, j}+u_{j, i}\right)+K\left(u_{j, i}-\varepsilon_{i j r} \phi_{r}\right), \\
& m_{i j}=\alpha \phi_{r, r} \delta_{i j}+\beta \phi_{i, j}+\gamma \phi_{j, i}, \\
& \lambda_{i}=\alpha_{0} \phi_{, i}^{*}, \\
& \lambda_{0} \nabla \phi^{*}+(\lambda+2 \mu+K) \nabla \nabla \cdot \boldsymbol{u}-(\mu+K) \nabla \times \nabla \times \boldsymbol{u}+K \nabla \times \boldsymbol{\phi}=\rho \ddot{\boldsymbol{u}}, \\
& (\alpha+\beta+\gamma) \nabla \nabla . \boldsymbol{\phi}-\gamma \nabla \times \nabla \times \boldsymbol{\phi}+K \nabla \times \boldsymbol{u}-2 K \boldsymbol{\phi}=\rho j \ddot{\boldsymbol{\phi}}, \\
& \alpha_{0} \nabla^{2} \phi^{*}-\frac{1}{3} \lambda_{1} \phi^{*}-\frac{\lambda_{0}}{3} \nabla \cdot \boldsymbol{u}=\frac{3}{2} \rho j \ddot{\phi}^{*},
\end{aligned}
$$

where

$$
\begin{array}{ll}
\lambda, \mu & =\text { Lame's constants, } \\
\alpha, \beta, \gamma, K & =\text { micropolar constants, } \\
\alpha_{o}, \lambda_{o}, \lambda_{1} & =\text { microstretch elastic constants, } \\
\rho & =\text { density } \\
j & =\text { micro-inertia, } \\
\boldsymbol{u} & =\text { displacement vector, } \\
\boldsymbol{\phi} & =\text { rotation vector } \\
\phi^{*} & =\text { the scalar microstretch, } \\
t_{i j} & =\text { force stress tensor } \\
m_{i j} & =\text { couple stress tensor, } \\
\lambda_{i} & =\text { first moment tensor, }
\end{array}
$$

and the dot denotes the partial derivative w.r.t. time.

\section{Formulation and solution}

The state of plane strain, parallel to the $x z$-plane, is defined by

$$
\begin{gathered}
u_{1}=u_{1}(x, z, t), u_{2}=0, u_{3}=u_{3}(x, z, t), \phi_{1}=\phi_{3}=0, \\
\phi_{2}=\phi_{2}(x, z, t), \phi^{*}=\phi^{*}(x, z, t)
\end{gathered}
$$

Therefore, the field equations (4)-(6) reduce to

$$
\begin{aligned}
& \lambda_{0} \frac{\partial \phi^{*}}{\partial x}+(\lambda+\mu)\left(\frac{\partial^{2} u_{1}}{\partial x^{2}}+\frac{\partial^{2} u_{3}}{\partial x \partial z}\right)+(\mu+K)\left(\frac{\partial^{2} u_{1}}{\partial x^{2}}+\frac{\partial^{2} u_{1}}{\partial z^{2}}\right) \\
& -K \frac{\partial \phi_{2}}{\partial z}=\rho \frac{\partial^{2} u_{1}}{\partial t^{2}} \\
& \lambda_{0} \frac{\partial \phi^{*}}{\partial z}+(\lambda+\mu)\left(\frac{\partial^{2} u_{1}}{\partial x \partial z}+\frac{\partial^{2} u_{3}}{\partial z^{2}}\right)+(\mu+K)\left(\frac{\partial^{2} u_{3}}{\partial x^{2}}+\frac{\partial^{2} u_{3}}{\partial z^{2}}\right) \\
& \quad+K \frac{\partial \phi_{2}}{\partial x}=\rho \frac{\partial^{2} u_{3}}{\partial t^{2}}, \\
& \gamma\left(\frac{\partial^{2} \phi_{2}}{\partial x^{2}}+\frac{\partial^{2} \phi_{2}}{\partial z^{2}}\right)-2 K \phi_{2}+K\left(\frac{\partial u_{1}}{\partial z}-\frac{\partial u_{3}}{\partial x}\right)=\rho j \frac{\partial^{2} \phi_{2}}{\partial t^{2}}
\end{aligned}
$$




$$
\alpha_{0}\left(\frac{\partial^{2} \phi^{*}}{\partial x^{2}}+\frac{\partial^{2} \phi^{*}}{\partial z^{2}}\right)-\frac{1}{3} \lambda_{1} \phi^{*}-\frac{\lambda_{0}}{3}\left(\frac{\partial u_{1}}{\partial x}+\frac{\partial u_{3}}{\partial z}\right)=\frac{3}{2} \rho j \frac{\partial^{2} \phi^{*}}{\partial t^{2}} .
$$

Introducing dimensionless quantities :

$$
\begin{aligned}
x^{\prime} & =\frac{x}{h}, z^{\prime}=\frac{z}{h}, u_{1}^{\prime}=\frac{\rho h \omega^{*^{2}} u_{1}}{\mu}, u_{3}^{\prime}=\frac{\rho h \omega^{*^{2}} u_{3}}{\mu}, \\
\phi_{2}^{\prime} & =\frac{\rho h^{2} \omega^{*^{2}} \phi_{2}}{\mu}, t^{\prime}=\frac{\mu}{\rho h^{2} \omega^{*}} t \\
\phi^{*^{\prime}} & =\frac{\rho h^{2} \omega^{*^{2}} \phi^{*}}{\mu}, t_{33}^{\prime}=\frac{1}{K} t_{33}, t_{31}^{\prime}=\frac{1}{K} t_{31}, \\
m_{32}^{\prime} & =\frac{1}{K h} m_{32}, \lambda_{3}^{\prime}=\frac{1}{K h} \lambda_{3}, \omega^{*^{2}}=\frac{K}{\rho j},
\end{aligned}
$$

where $h$ is the standard length.

Using quantities (12) in (8)-(11) and suppressing the dashes, we obtain

$$
\begin{aligned}
& S_{0} \frac{\partial \phi^{*}}{\partial x}+\frac{\partial^{2} u_{1}}{\partial x^{2}}+\left(1-a^{2}\right) \frac{\partial^{2} u_{3}}{\partial x \partial z}+a^{2} \frac{\partial^{2} u_{1}}{\partial z^{2}}-S_{4}^{*} \frac{\partial \phi^{*}}{\partial z}=\frac{1}{\left(S_{1}+S_{2}\right)} \frac{\partial^{2} u_{1}}{\partial t^{2}}, \\
& S_{0} \frac{\partial \phi^{*}}{\partial z}+\left(1-a^{2}\right) \frac{\partial^{2} u_{1}}{\partial x \partial z}+a^{2} \frac{\partial^{2} u_{3}}{\partial x^{2}}+\frac{\partial^{2} u_{3}}{\partial z^{2}}+S_{4}^{*} \frac{\partial \phi_{2}}{\partial x}=\frac{1}{\left(S_{1}+S_{2}\right)} \frac{\partial^{2} u_{3}}{\partial t^{2}}, \\
& \left(\frac{\partial^{2} \phi_{2}}{\partial x^{2}}+\frac{\partial^{2} \phi_{2}}{\partial z^{2}}\right)-\frac{2 K h^{2} \phi_{2}}{\gamma}+\frac{K h^{2}}{\gamma}\left(\frac{\partial u_{1}}{\partial z}-\frac{\partial u_{3}}{\partial x}\right)=\frac{1}{S_{4}} \frac{\partial^{2} \phi_{2}}{\partial t^{2}}, \\
& \left(\frac{\partial^{2} \phi^{*}}{\partial x^{2}}+\frac{\partial^{2} \phi^{*}}{\partial z^{2}}\right)-S_{5} \phi^{*}-S_{6}\left(\frac{\partial u_{1}}{\partial x}+\frac{\partial u_{3}}{\partial z}\right)=S_{7} \frac{\partial^{2} \phi^{*}}{\partial t^{2}}
\end{aligned}
$$

where

$$
\begin{aligned}
& S_{0}=\frac{\lambda_{0}}{\lambda+2 \mu+K}, S_{1}=\frac{\rho h^{2}(\lambda+\mu) \omega^{*^{2}}}{\mu^{2}}, S_{2}=\frac{\rho h^{2}(\lambda+K) \omega^{*^{2}}}{\mu^{2}} \\
& S_{3}=\frac{\rho h^{2} K \omega^{*^{2}}}{\mu^{2}}, S_{4}=\frac{\gamma \rho h^{2} \omega^{*^{2}}}{j \mu^{2}}, a^{2}=\frac{S_{3}}{S_{1}+S_{2}}, S_{4}^{*}=\frac{S_{3}}{S_{1}+S_{2}} \\
& S_{5}=\frac{\lambda_{1} h^{2}}{3 \alpha_{0}}, S_{6}=\frac{\lambda_{0} h^{2}}{3 \alpha_{0}}, S_{7}=\frac{3 j \mu^{2}}{2 \rho \alpha_{0} h^{2} \omega^{*^{2}}}
\end{aligned}
$$

Applying the Laplace transform w.r.t. time defined by

$$
\begin{aligned}
& \left\{\boldsymbol{u}_{1}(x, z, p), \boldsymbol{u}_{3}(x, z, p), \overline{\boldsymbol{\phi}}_{2}(x, z, p), \overline{\boldsymbol{\phi}}^{*}(x, z, p)\right\} \\
& \quad=\int_{-\infty}^{\infty}\left\{u_{1}(x, z, t), u_{3}(x, z, t), \phi_{2}(x, z, t), \phi^{*}(x, z, t)\right\} \exp (-p t) \mathrm{d} t,
\end{aligned}
$$

and then Fourier transforms w.r.t. ' $x$ ', defined by

$$
\begin{aligned}
& \left\{\tilde{u}_{1}(\xi, z, p), \tilde{u}_{3}(\xi, z, p), \tilde{\phi}_{2}(\xi, z, p), \tilde{\phi}^{*}(\xi, z, p)\right\} \\
& \quad=\int_{-\infty}^{\infty}\left\{\boldsymbol{u}_{1}(x, z, p), \boldsymbol{u}_{3}(x, z, p), \overline{\boldsymbol{\phi}}_{2}(x, z, p), \overline{\boldsymbol{\phi}}^{*}(x, z, p)\right\} \exp (-i \xi x) \mathrm{d} x
\end{aligned}
$$


on (13)-(16), we obtain

$$
\begin{aligned}
\tilde{u}_{1}^{\prime \prime} & =\frac{1}{a^{2}}\left(\xi^{2}+\frac{p^{2}}{S_{1}+S_{2}}\right) \tilde{u}_{1}-\frac{i \xi\left(1-a^{2}\right)}{a^{2}} \tilde{u}_{3}^{\prime}+\frac{S_{4}^{*}}{a^{2}} \tilde{\phi}_{2}^{\prime}-\frac{i \xi S_{0}}{a^{2}} \tilde{\phi}^{*}, \\
\tilde{u}_{3}^{\prime \prime} & =\left(a^{2} \xi^{2}+\frac{p^{2}}{S_{1}+S_{2}}\right) \tilde{u}_{3}-\left(1-a^{2}\right) i \xi \tilde{u}_{1}^{\prime}-i \xi S_{4}^{*} \tilde{\phi}_{2}-S_{0} \tilde{\phi}^{*}, \\
\tilde{\phi}_{2}^{\prime \prime} & =\frac{K h^{2}}{\gamma} \tilde{u}_{1}^{\prime}+\frac{i \xi K h^{2}}{\gamma} \tilde{u}_{3}+\left(\xi^{2}+\frac{2 K h^{2}}{\gamma}+\frac{p^{2}}{S_{4}}\right) \tilde{\phi}_{2} \\
\text { and } \tilde{\phi}^{* \prime} & =i S_{6} \xi \tilde{u}_{1}+S_{6} \tilde{u}_{3}^{\prime}+\left(p^{2} S_{7}+\xi^{2}+S_{5}\right) \tilde{\phi}^{*}
\end{aligned}
$$

The system of equations (20)-(23) can be written as

$$
\frac{\mathrm{d}}{\mathrm{d} z} W(\xi, z, p)=A(\xi, p) W(\xi, z, p)
$$

where

$$
\begin{aligned}
& W=\left[\begin{array}{c}
U \\
U^{\prime}
\end{array}\right], \quad A=\left[\begin{array}{cc}
O & I \\
A_{2} & A_{1}
\end{array}\right], \quad U=\left[\begin{array}{c}
\tilde{u}_{1} \\
\tilde{u}_{3} \\
\tilde{\phi}_{2} \\
\tilde{\phi}^{*}
\end{array}\right] \\
& O=\left[\begin{array}{llll}
0 & 0 & 0 & 0 \\
0 & 0 & 0 & 0 \\
0 & 0 & 0 & 0 \\
0 & 0 & 0 & 0
\end{array}\right], \quad I=\left[\begin{array}{llll}
1 & 0 & 0 & 0 \\
0 & 1 & 0 & 0 \\
0 & 0 & 1 & 0 \\
0 & 0 & 0 & 1
\end{array}\right] \\
& A_{1}=\left[\begin{array}{cccc}
0 & \frac{-\left(1-a^{2}\right) i \xi}{a^{2}} & \frac{S_{4}^{*}}{a^{2}} & 0 \\
-\left(1-a^{2}\right) i \xi & 0 & 0 & -S_{0} \\
\frac{-K h^{2}}{\gamma} & 0 & 0 & 0 \\
0 & S_{6} & 0 & 0
\end{array}\right] \\
& A_{2}=\left[\begin{array}{cccc}
\frac{1}{a^{2}}\left(\xi^{2}+\frac{p^{2}}{S_{1}+S_{2}}\right) & 0 & 0 & \frac{-i \xi S_{0}}{a^{2}} \\
0 & a^{2} \xi^{2}+\frac{p^{2}}{S_{1}+S_{2}} & -i S_{4}^{*} \xi & 0 \\
0 & \frac{i K \xi h^{2}}{\gamma} & \xi^{2}+\frac{2 K h^{2}}{\gamma}+\frac{p^{2}}{S_{4}} & 0 \\
i \xi S_{6} & 0 & 0 & \left(\xi^{2}+S_{5}+p^{2} S_{7}\right)
\end{array}\right] \text {. }
\end{aligned}
$$

To solve (24), we take

$$
W(\xi, z, p)=X(\xi, p) \exp (q z)
$$

so that

$$
A(\xi, p) W(\xi, z, p)=q W(\xi, z, p)
$$

which leads to an eigenvalue problem. The characteristic equation corresponding to the matrix $A$ is given by

$$
\operatorname{det}(A-q I)=0,
$$


which on expansion provides us

$$
q^{8}-\lambda_{1} q^{6}+\lambda_{2} q^{4}-\lambda_{3} q^{2}+\lambda_{4}=0
$$

where

$$
\begin{aligned}
& \lambda_{1}=4 \xi^{2}+\frac{p^{2}}{S_{1}+S_{2}}+\frac{2 K h^{2}}{\gamma}+\frac{p^{2}}{S_{4}}+\frac{p^{2}}{a^{2}\left(S_{1}+S_{2}\right)}+S_{5}+p^{2} S_{7} \\
& -S_{0} S_{6}-\frac{S_{4}^{*} K h^{2}}{\gamma a^{2}} \\
& \lambda_{2}=\left(\xi^{2}+\frac{p^{2}}{S_{1}+S_{2}}\right)\left(\xi^{2}+\frac{2 K h^{2}}{\gamma}+\frac{p^{2}}{S_{4}}\right)+\left(\xi^{2}+\frac{2 K h^{2}}{\gamma}+\frac{p^{2}}{S_{4}}\right) \\
& \left(\frac{p^{2} / a^{2}}{S_{1}+S_{2}}+\xi^{2}\right)+\left(\xi^{2}+\frac{p^{2}}{S_{1}+S_{2}}\right)\left(\frac{p^{2} / a^{2}}{S_{1}+S_{2}}+\xi^{2}\right) \\
& +\left(\xi^{2}+\frac{p^{2}}{S_{1}+S_{2}}\right)\left(\xi^{2}+S_{5}+p^{2} S_{7}\right)+\left(\xi^{2}+\frac{2 K h^{2}}{\gamma}+\frac{p^{2}}{S_{4}}\right) \\
& \left(\xi^{2}+S_{5}+p^{2} S_{7}\right)+\left(\frac{p^{2} / a^{2}}{S_{1}+S_{2}}+\xi^{2}\right)\left(\xi^{2}+S_{5}+p^{2} S_{7}\right)+\frac{S_{0} S_{4}^{*} S_{6} K h^{2}}{\gamma a^{2}} \\
& -S_{0} S_{6}\left(3 \xi^{2}+\frac{p^{2}}{S_{4}}+\frac{2 K h^{2}}{\gamma}+\frac{p^{2} / a^{2}}{S_{1}+S_{2}}\right) \\
& -\frac{S_{4}^{*} K h^{2}}{\gamma a^{2}}\left(3 \xi^{2}+\frac{p^{2}}{S_{1}+S_{2}}+S_{5}+p^{2} S_{7}\right) \\
& \lambda_{3}=\left(\xi^{2}+\frac{p^{2}}{S_{1}+S_{2}}\right)\left(\xi^{2}+\frac{2 K h^{2}}{\gamma}+\frac{p^{2}}{S_{4}}\right)\left(\frac{p^{2} / a^{2}}{S_{1}+S_{2}}+\xi^{2}\right) \\
& +\left(\xi^{2}+\frac{2 K h^{2}}{\gamma}+\frac{p^{2}}{S_{4}}\right)\left(\frac{p^{2} / a^{2}}{S_{1}+S_{2}}+\xi^{2}\right)\left(\xi^{2}+S_{5}+p^{2} S_{7}\right) \\
& +\left(\frac{p^{2} / a^{2}}{S_{1}+S_{2}}+\xi^{2}\right)\left(\xi^{2}+S_{5}+p^{2} S_{7}\right)\left(\xi^{2}+\frac{p^{2}}{S_{1}+S_{2}}\right) \\
& +\left(\xi^{2}+S_{5}+p^{2} S_{7}\right)\left(\xi^{2}+\frac{p^{2}}{S_{1}+S_{2}}\right)\left(\xi^{2}+\frac{2 K h^{2}}{\gamma}+\frac{p^{2}}{S_{4}}\right) \\
& -\frac{S_{4}^{*} K h^{2}}{\gamma a^{2}}\left\{\left(\xi^{2}+S_{5}+p^{2} S_{7}\right)\left(2 \xi^{2}+\frac{p^{2}}{S_{1}+S_{2}}\right)+\xi^{2}\left(\xi^{2}+\frac{p^{2}}{S_{1}+S_{2}}\right)\right\} \\
& +\frac{2 S_{0} S_{4}^{*} S_{6} K \xi^{2} h^{2}}{\gamma a^{2}}-S_{0} S_{6}\left\{\left(\xi^{2}+\frac{2 K h^{2}}{\gamma}+\frac{p^{2}}{S_{4}}\right)\left(\frac{p^{2} / a^{2}}{S_{1}+S_{2}}+\xi^{2}\right)\right. \\
& \left.+\xi^{2}\left(\frac{p^{2} / a^{2}}{S_{1}+S_{2}}+\xi^{2}\right)+\xi^{2}\left(\xi^{2}+\frac{2 K h^{2}}{\gamma}+\frac{p^{2}}{S_{4}}\right)\right\}
\end{aligned}
$$


and

$$
\begin{aligned}
\lambda_{4}= & \left(\xi^{2}+\frac{p^{2}}{S_{1}+S_{2}}\right)\left(\xi^{2}+\frac{2 K h^{2}}{\gamma}+\frac{p^{2}}{S_{4}}\right)\left(\frac{p^{2} / a^{2}}{S_{1}+S_{2}}+\xi^{2}\right)\left(\xi^{2}+S_{5}+p^{2} S_{7}\right) \\
& +\frac{S_{0} S_{4}^{*} S_{6} K h^{2} \xi^{4}}{\gamma a^{2}}-S_{0} S_{6} \xi^{2}\left(\frac{p^{2} / a^{2}}{S_{1}+S_{2}}+\xi^{2}\right)\left(\xi^{2}+\frac{2 K h^{2}}{\gamma}+\frac{p^{2}}{S_{4}}\right) \\
& -\frac{S_{4}^{*} K h^{2} \xi^{2}}{\gamma a^{2}}\left(\xi^{2}+S_{5}+p^{2} S_{7}\right)\left(\xi^{2}+\frac{p^{2}}{S_{1}+S_{2}}\right) .
\end{aligned}
$$

The eigenvalues of the matrix $A$ are characteristic roots of (29) assuming that real parts of $q_{s}$ are positive. The vectors $\boldsymbol{X}(\xi, p)$ corresponding to the eigenvalues $q_{s}$ can be determined by solving the homogeneous equations

$$
[A-q I] \boldsymbol{X}(\xi, p)=0 .
$$

The set of eigenvectors $\boldsymbol{X}_{s}(\xi, p), s=1,2,3, \ldots, 8$ may be defined as

$$
X_{s}(\xi, p)=\left[\begin{array}{l}
X_{s 1}(\xi, p) \\
X_{s 2}(\xi, p)
\end{array}\right]
$$

where

$$
\begin{aligned}
X_{s 1}(\xi, p)= & {\left[\begin{array}{c}
a_{s} q_{s} \\
b_{s} \\
-\xi \\
c_{s}
\end{array}\right], X_{s 2}(\xi, p)=\left[\begin{array}{c}
a_{s} q_{s}^{2} \\
b_{s} q_{s} \\
-\xi q_{s} \\
c_{s} q_{s}
\end{array}\right], q=q_{s} ; s=1,2,3,4 } \\
X_{j 1}(\xi, p)= & {\left[\begin{array}{c}
-a_{s} q_{s} \\
b_{s} \\
-\xi \\
c_{s}
\end{array}\right], X_{j 2}(\xi, p)=\left[\begin{array}{c}
a_{s} q_{s}^{2} \\
-b_{s} q_{s} \\
\xi q_{s} \\
-c_{s} q_{s}
\end{array}\right], j=s+4, q=-q_{s} } \\
s= & 1,2,3,4
\end{aligned}
$$

and

$$
\begin{aligned}
a_{s}= & \left(\xi^{2}+\frac{2 K h^{2}}{\gamma}+\frac{p^{2}}{S_{4}}-q_{s}^{2}\right)\left\{\xi\left(1-a^{2}\right)\left(\xi^{2}+S_{5}+p^{2} S_{7}-q_{s}^{2}\right)-\xi S_{0} S_{6}\right\} \\
& \left.+\frac{K \xi h^{2} S_{4}^{*}}{\gamma}\left(\xi^{2}+S_{5}+p^{2} S_{7}-q_{s}^{2}\right)\right] / \Delta \\
b_{s}=-i & {\left[( \xi ^ { 2 } + \frac { 2 K h ^ { 2 } } { \gamma } + \frac { p ^ { 2 } } { S _ { 4 } } - q _ { s } ^ { 2 } ) \left\{\left(\xi^{2}+S_{5}+p^{2} S_{7}-q_{s}^{2}\right)\right.\right.} \\
& \left.\times\left(\xi^{2}+\frac{p^{2}}{S_{1}+S_{2}}-a^{2} q_{s}^{2}\right)-\xi^{2} S_{0} S_{6}\right\}+\frac{K S_{4}^{*} h^{2} q_{s}^{2}}{\gamma} \\
& \left.\times\left(\xi^{2}+S_{5}+p^{2} S_{7}-q_{s}^{2}\right)\right] / \Delta, \\
c_{s}= & q_{s} S_{6}\left(b_{s}+i \xi a_{s}\right) /\left\{q_{s}^{2}-\left(\xi^{2}+S_{5}+p^{2} S_{7}\right)\right\} \\
\Delta= & \frac{K h^{2}}{\gamma}\left\{\left(\xi^{2}+S_{5}+p^{2} S_{7}-q_{s}^{2}\right)\left(\xi^{2}+\frac{p^{2}}{S_{1}+S_{2}}-q_{s}^{2}\right)+\left(q_{s}^{2}-\xi^{2}\right) S_{0} S_{6}\right\} \\
& s=1,2,3,4
\end{aligned}
$$


The solution of (26) is given by (c.f. Sharma \& Chand 1992),

$$
W(\xi, z, p)=\sum_{s=1}^{4}\left[B_{s} X_{s}(\xi, p) \exp \left(q_{s}, z\right)+B_{s+4} X_{s+4}(\xi, p) \exp (-q, z)\right]
$$

where $B_{s}(s=1,2,3, \ldots, 8)$ are arbitrary constants.

Equation (42) represents the solution of the general problem in the plane strain case of microstretch elastic solid by employing the eigenvalue approach and therefore can be applied to a broad class of problems in the domain of Laplace and Fourier transforms.

\section{Application}

We consider an infinite microstretch elastic space in which a concentrated force of magnitude $F=-F_{0} \delta(x) \delta(t)$, where $F_{0}$ is the magnitude of the force, is acting in the direction of the $z$-axis at the origin of the cartesian co-ordinate system. The problem is plane strain w.r.t. the $x z$-plane and the boundary conditions on the plane $z=0$ are given as,

$$
\begin{aligned}
& u_{1}\left(x, 0^{+}, t\right)-u_{1}\left(x, 0^{-}, t\right)=0, \quad u_{3}\left(x, 0^{+}, t\right)-u_{3}\left(x, 0^{-}, t\right)=0, \\
& \phi_{2}\left(x, 0^{+}, t\right)-\phi_{2}\left(x, 0^{-}, t\right)=0, \quad \phi^{*}\left(x, 0^{+}, t\right)-\phi^{*}\left(x, 0^{-}, t\right)=0, \\
& m_{32}\left(x, 0^{+}, t\right)-m_{32}\left(x, 0^{-}, t\right)=0, \quad t_{31}\left(x, 0^{+}, t\right)-t_{31}\left(x, 0^{-}, t\right)=0, \\
& t_{33}\left(x, 0^{+}, t\right)-t_{33}\left(x, 0^{-}, t\right)=-F_{o} \delta(x) \delta(t), \quad \lambda_{3}\left(x, 0^{+}, t\right)-\lambda_{3}\left(x, 0^{-}, t\right)=0 .
\end{aligned}
$$

Applying the Laplace and Fourier transforms to (43)-(46), we get,

$$
\begin{aligned}
& \tilde{u}_{1}\left(\xi, 0^{+}, p\right)-\tilde{u}_{1}\left(\xi, 0^{-}, p\right)=0, \quad \tilde{u}_{3}\left(\xi, 0^{+}, p\right)-\tilde{u}_{3}\left(\xi, 0^{-}, p\right)=0, \\
& \tilde{\phi}_{2}\left(\xi, 0^{+}, p\right)-\tilde{\phi}_{2}\left(\xi, 0^{-}, p\right)=0, \quad \tilde{\phi}^{*}\left(\xi, 0^{+}, p\right)-\tilde{\phi}^{*}\left(\xi, 0^{-}, p\right)=0, \\
& \tilde{m}_{32}\left(\xi, 0^{+}, p\right)-\tilde{m}_{32}\left(\xi, 0^{-}, p\right)=0, \quad \tilde{t}_{31}\left(\xi, 0^{+}, p\right)-\tilde{t}_{31}\left(\xi, 0^{-}, p\right)=0, \\
& \tilde{t}_{33}\left(\xi, 0^{+}, p\right)-\tilde{t}_{33}\left(\xi, 0^{-}, p\right)=-F_{o}, \quad \tilde{\lambda}_{3}\left(\xi, 0^{+}, p\right)-\tilde{\lambda}_{3}\left(\xi, 0^{-}, p\right)=0 .
\end{aligned}
$$

The transformed displacements, microrotation, microstretch, stresses and first moment are given for $z \geq 0$,

$$
\begin{aligned}
\tilde{u}_{1}(\xi, z, p)= & -\left[a_{1} q_{1} B_{5} \exp \left(-q_{1} z\right)+a_{2} q_{2} B_{6} \exp \left(-q_{2} z\right)+a_{3} q_{3} B_{7} \exp \left(-q_{3} z\right)\right. \\
& \left.+a_{4} q_{4} B_{8} \exp \left(-q_{4} z\right)\right] \\
\tilde{u}_{3}(\xi, z, p)= & b_{1} B_{5} \exp \left(-q_{1} z\right)+b_{2} B_{6} \exp \left(-q_{2} z\right)+b_{3} B_{7} \exp \left(-q_{3} z\right) \\
& +b_{4} B_{8} \exp \left(-q_{4} z\right) \\
\tilde{\phi}_{2}(\xi, z, p)= & -\xi\left[B_{5} \exp \left(-q_{1} z\right)+B_{6} \exp \left(-q_{2} z\right)+B_{7} \exp \left(-q_{3} z\right)\right. \\
& \left.+B_{8} \exp \left(-q_{4} z\right)\right] \\
\tilde{\phi}^{*}(\xi, z, p)= & c_{1} B_{5} \exp \left(-q_{1} z\right)+c_{2} B_{6} \exp \left(-q_{2} z\right)+c_{3} B_{7} \exp \left(-q_{3} z\right) \\
& +c_{4} B_{8} \exp \left(-q_{4} z\right) \\
\tilde{m}_{32}(\xi, z, p)= & \xi S_{8}\left[q_{1} B_{5} \exp \left(-q_{1} z\right)+q_{2} B_{6} \exp \left(-q_{2} z\right)+q_{3} B_{7} \exp \left(-q_{3} z\right)\right. \\
& \left.+q_{4} B_{8} \exp \left(-q_{4} z\right)\right]
\end{aligned}
$$




$$
\begin{aligned}
\tilde{t}_{31}(\xi, z, p)= & \left(a_{1} S_{9} q_{1}^{2}+i \xi b_{1} S_{10}+\xi S_{11}\right) B_{5} \exp \left(-q_{1} z\right) \\
& +\left(a_{2} S_{9} q_{2}^{2}+i \xi b_{2} S_{10}+\xi S_{11}\right) B_{6} \exp \left(-q_{2} z\right) \\
& +\left(a_{3} S_{9} q_{3}^{2}+i \xi b_{3} S_{10}+\xi S_{11}\right) B_{7} \exp \left(-q_{3} z\right) \\
& +\left(a_{4} S_{9} q_{4}^{2}+i \xi b_{4} S_{10}+\xi S_{11}\right) B_{8} \exp \left(-q_{4} z\right), \\
\tilde{t}_{33}(\xi, z, p)= & \left(c_{1} S_{12}-b_{1} q_{1} S_{13}-i \xi a_{1} q_{1} S_{14}\right) B_{5} \exp \left(-q_{1} z\right) \\
& +\left(c_{2} S_{12}-b_{2} q_{2} S_{14}-i \xi a_{2} q_{2} S_{14}\right) B_{6} \exp \left(-q_{2} z\right) \\
& +\left(c_{3} S_{12}-b_{3} q_{3} S_{14}-i \xi a_{3} q_{3} S_{14}\right) B_{7} \exp \left(-q_{3} z\right) \\
& +\left(c_{4} S_{12}-b_{4} q_{4} S_{14}-i \xi a_{4} q_{4} S_{14}\right) B_{8} \exp \left(-q_{4} z\right), \\
\tilde{\lambda}_{3}(\xi, z, p)= & -S_{15}\left[c_{1} q_{1} B_{5} \exp \left(-q_{1} z\right)+c_{2} q_{2} B_{6} \exp \left(-q_{2} z\right)\right. \\
& \left.+c_{3} q_{3} B_{7} \exp \left(-q_{3} z\right)+c_{4} q_{4} B_{8} \exp \left(-q_{4} z\right)\right] .
\end{aligned}
$$

Similary for $z \leq 0$

$$
\begin{aligned}
\tilde{u}_{1}(\xi, z, p)= & a_{1} q_{1} B_{1} \exp \left(q_{1} z\right)+a_{2} q_{2} B_{2} \exp \left(q_{2} z\right)+a_{3} q_{3} B_{3} \exp \left(q_{3} z\right) \\
& +a_{4} q_{4} B_{4} \exp \left(q_{4} z\right) \\
\tilde{u}_{3}(\xi, z, p)= & b_{1} B_{1} \exp \left(q_{1} z\right)+b_{2} B_{2} \exp \left(q_{2} z\right)+b_{3} B_{3} \exp \left(q_{3} z\right) \\
& +b_{4} B_{4} \exp \left(q_{4} z\right) \\
\tilde{\phi}_{2}(\xi, z, p)= & -\xi\left[B_{1} \exp \left(q_{1} z\right)+B_{2} \exp \left(q_{2} z\right)+B_{3} \exp \left(q_{3} z\right)+B_{4} \exp \left(q_{4} z\right)\right] \\
\tilde{\phi}^{*}(\xi, z, p)= & c_{1} B_{1} \exp \left(q_{1} z\right)+c_{2} B_{2} \exp \left(q_{2} z\right)+c_{3} B_{3} \exp \left(q_{3} z\right) \\
& +c_{4} B_{4} \exp \left(q_{4} z\right) \\
\tilde{m}_{32}(\xi, z, p)= & -\xi S_{8}\left[q_{1} B_{1} \exp \left(q_{1} z\right)+q_{2} B_{2} \exp \left(q_{2} z\right)+q_{3} B_{3} \exp \left(q_{3} z\right)\right. \\
& \left.+q_{4} B_{4} \exp \left(q_{4} z\right)\right] \\
\tilde{t}_{31}(\xi, z, p)= & \left(a_{1} S_{9} q_{1}^{2}+i \xi b_{1} S_{10}+\xi S_{11}\right) B_{1} \exp \left(q_{1} z\right) \\
& +\left(a_{2} S_{9} q_{2}^{2}+i \xi b_{2} S_{10}+\xi S_{11}\right) B_{2} \exp \left(q_{2} z\right) \\
& +\left(a_{3} S_{9} q_{3}^{2}+i \xi b_{3} S_{10}+\xi S_{11}\right) B_{3} \exp \left(q_{3} z\right) \\
& +\left(a_{4} S_{9} q_{4}^{2}+i \xi b_{4} S_{10}+\xi S_{11}\right) B_{4} \exp \left(q_{4} z\right) \\
\tilde{t}_{33}(\xi, z, p)= & \left(c_{1} S_{12}+b_{1} q_{1} S_{13}+i \xi a_{1} q_{1} S_{14}\right) B_{1} \exp \left(q_{1} z\right) \\
& +\left(c_{2} S_{12}+b_{2} q_{2} S_{13}+i \xi a_{2} q_{2} S_{14}\right) B_{2} \exp \left(q_{2} z\right) \\
& +\left(c_{3} S_{12}+b_{3} q_{3} S_{13}+i \xi a_{3} q_{3} S_{14}\right) B_{3} \exp \left(q_{3} z\right) \\
& +\left(c_{4} S_{12}+b_{4} q_{4} S_{13}+i \xi a_{4} q_{4} S_{14}\right) B_{4} \exp \left(q_{4} z\right) \\
& S_{15}\left[c_{1} q_{1} B_{1} \exp \left(q_{1} z\right)+c_{2} q_{2} B_{2} \exp \left(q_{2} z\right)+c_{3} q_{3} B_{3} \exp \left(q_{3} z\right)\right. \\
& \left.+c_{4} q_{4} B_{4} \exp \left(q_{4} z\right)\right] \\
\tilde{\lambda}_{3}(\xi, z, p) &
\end{aligned}
$$

where

$$
\begin{aligned}
S_{8} & =\frac{\gamma \mu}{K \rho h^{4} \omega^{*^{2}}}, S_{9}=\frac{\mu(\mu+K)}{\rho K h^{2} \omega^{*^{2}}}, S_{10}=\frac{\mu^{2}}{\rho K h^{2} \omega^{*^{2}}}, S_{11}=\frac{\mu}{\rho h^{2} \omega^{*^{2}}}, \\
S_{12} & =\frac{\lambda_{0} \mu}{K \rho h^{2} \omega^{*^{2}}}, S_{13}=\frac{\mu(\lambda+2 \mu+K)}{K \rho h^{2} \omega^{*^{2}}}, S_{14}=\frac{\lambda \mu}{K \rho h^{2} \omega^{*^{2}}}, S_{15}=\frac{\alpha_{0} \mu}{K \rho h^{4} \omega^{*^{2}}} .
\end{aligned}
$$


Using conditions (47)-(50) in (51)-(66), we obtain

$$
\begin{aligned}
& a_{1} q_{1}\left(B_{1}+B_{5}\right)+a_{2} q_{2}\left(B_{2}+B_{6}\right)+a_{3} q_{3}\left(B_{3}+B_{7}\right)+a_{4} q_{4}\left(B_{4}+B_{8}\right)=0, \\
& b_{1}\left(B_{1}-B_{5}\right)+b_{2}\left(B_{2}-B_{6}\right)+b_{3}\left(B_{3}-B_{7}\right)+b_{4}\left(B_{4}-B_{8}\right)=0, \\
& \left(B_{1}-B_{5}\right)+\left(B_{2}-B_{6}\right)+\left(B_{3}-B_{7}\right)+\left(B_{4}-B_{8}\right)=0, \\
& q_{1}\left(B_{1}+B_{5}\right)+q_{2}\left(B_{2}+B_{6}\right)+q_{3}\left(B_{3}+B_{7}\right)+q_{4}\left(B_{4}+B_{8}\right)=0 \\
& c_{1}\left(B_{1}-B_{5}\right)+c_{2}\left(B_{2}-B_{6}\right)+c_{3}\left(B_{3}-B_{7}\right)+c_{4}\left(B_{4}-B_{8}\right)=0 \\
& \left(a_{1} q_{1}^{2} S_{9}+i \xi b_{1} S_{10}+\xi S_{11}\right)\left(B_{1}-B_{5}\right)+\left(a_{2} q_{2}^{2} S_{9}+i \xi b_{2} S_{10}+\xi S_{11}\right)\left(B_{2}-B_{6}\right) \\
& \quad+\left(a_{3} q_{3}^{2} S_{9}+i \xi b_{3} S_{10}+\xi S_{11}\right)\left(B_{3}-B_{7}\right) \\
& \quad+\left(a_{4} q_{4}^{2} S_{9}+i \xi b_{4} S_{10}+\xi S_{11}\right)\left(B_{4}-B_{8}\right)=0 \\
& \quad q_{1}\left(b_{1} S_{13}+i \xi a_{1} S_{14}\right)\left(B_{1}+B_{5}\right)+q_{2}\left(b_{2} S_{13}+i \xi a_{2} S_{14}\right)\left(B_{2}+B_{6}\right) \\
& \quad+q_{3}\left(b_{3} S_{13}+i \xi a_{3} S_{14}\right)\left(B_{3}+B_{7}\right)+q_{4}\left(b_{4} S_{13}+i \xi a_{4} S_{14}\right)\left(B_{4}+B_{8}\right)=F_{0} \\
& \quad c_{1} q_{1}\left(B_{1}+B_{5}\right)+c_{2} q_{2}\left(B_{2}+B_{6}\right)+c_{3} q_{3}\left(B_{3}+B_{7}\right)+c_{4} q_{4}\left(B_{4}+B_{8}\right)=0
\end{aligned}
$$

Solving the system of equations (68)-(75), we obtain

$$
\begin{aligned}
& B_{1}=B_{5}=F_{o}\left[c_{2}\left(a_{3}-a_{4}\right)+c_{3}\left(a_{4}-a_{2}\right)+c_{4}\left(a_{2}-a_{3}\right)\right] / 2 q_{1} \Delta_{1}, \\
& B_{2}=B_{6}=F_{o}\left[c_{1}\left(a_{4}-a_{3}\right)+c_{3}\left(a_{1}-a_{4}\right)+c_{4}\left(a_{3}-a_{1}\right)\right] / 2 q_{2} \Delta_{1}, \\
& B_{3}=B_{7}=F_{o}\left[c_{1}\left(a_{2}-a_{4}\right)+c_{2}\left(a_{4}-a_{1}\right)+c_{4}\left(a_{1}-a_{2}\right)\right] / 2 q_{3} \Delta_{1}, \\
& B_{4}=B_{8}=F_{o}\left[c_{1}\left(a_{3}-a_{2}\right)+c_{2}\left(a_{1}-a_{3}\right)+c_{3}\left(a_{2}-a_{1}\right)\right] / 2 q_{4} \Delta_{1},
\end{aligned}
$$

where

$$
\begin{aligned}
\Delta_{1}= & S_{13}\left[c_{1}\left\{\left(a_{2} b_{3}-a_{3} b_{2}\right)+\left(a_{3} b_{4}-a_{4} b_{3}\right)+\left(a_{4} b_{2}-a_{2} b_{4}\right)\right\}\right. \\
& +c_{2}\left\{\left(a_{3} b_{1}-a_{1} b_{3}\right)+\left(a_{1} b_{4}-a_{4} b_{1}\right)+\left(a_{4} b_{3}-a_{3} b_{4}\right)\right\} \\
& +c_{3}\left\{\left(a_{1} b_{2}-a_{2} b_{1}\right)+\left(a_{4} b_{1}-a_{1} b_{4}\right)+\left(a_{2} b_{4}-a_{4} b_{2}\right)\right\} \\
& \left.+c_{4}\left\{\left(a_{2} b_{1}-a_{1} b_{2}\right)+\left(a_{1} b_{3}-a_{3} b_{1}\right)+\left(a_{3} b_{2}-a_{2} b_{3}\right)\right\}\right] .
\end{aligned}
$$

Thus functions $\tilde{u}_{1}, \tilde{u}_{3}, \tilde{\phi}_{2}, \tilde{\phi}^{*}, \tilde{m}_{32}, \tilde{t}_{31}, \tilde{t}_{33}$ and $\tilde{\lambda}_{3}$ have been determined in the transform domain and these enable us to find the displacements, microrotation, stresses and first moment.

\section{Special case}

By neglecting stretch effect in (30)-(32) and (51)-(66), we get normal displacement, couple stress and normal force stress in transformed domain for micropolar elastic medium as

$$
\begin{aligned}
\tilde{u}_{3}(\xi, z, p)= & \left.b_{1} B_{4} \exp \left(-q_{1} z\right)\right)+b_{2} B_{5} \exp \left(-q_{2} z\right)+b_{3} B_{6} \exp \left(-q_{3} z\right) \\
\tilde{m}_{32}(\xi, z, p)= & \left.\xi S_{8}\left\{q_{1} B_{4} \exp \left(-q_{1} z\right)\right)+q_{2} B_{5} \exp \left(-q_{2} z\right)+q_{3} B_{6} \exp \left(-q_{3} z\right)\right\} \\
\tilde{t}_{33}(\xi, z, p)= & -\left\{\left(b_{1} q_{1} S_{13}+i \xi a_{1} q_{1} S_{14}\right) B_{4} \exp \left(-q_{1} z\right)\right. \\
& +\left(b_{2} q_{2} S_{13}+i \xi a_{2} q_{2} S_{14}\right) B_{5} \exp \left(-q_{2} z\right) \\
& +\left(b_{3} q_{3} S_{13}+i \xi a_{3} q_{3} S_{14}\right) B_{6} \exp \left(-q_{3} z\right)
\end{aligned}
$$




$$
\begin{aligned}
& \text { where } a_{s}=\left[\xi\left(a^{2}-1\right)\left\{\left(\xi^{2}+\frac{2 K h^{2}}{\gamma}+\frac{p^{2}}{S_{4}}\right)-q_{s}^{2}\right\}-\frac{K \xi h^{2} S_{4}^{*}}{\gamma}\right] / \Delta_{0}, \\
& \qquad \begin{aligned}
b_{s}= & i\left[\left(\xi^{2}+\frac{p^{2}}{S_{1}+S_{2}}\right)\left(\xi^{2}+\frac{2 K h^{2}}{\gamma}+\frac{p^{2}}{S_{4}}\right)\right. \\
& +a^{2} q_{s}^{2}\left\{q_{s}^{2}-\left(\xi^{2}+\frac{2 K h^{2}}{\gamma}+\frac{p^{2}}{S_{4}}\right)\right\} \\
& \left.-\left(\xi^{2}+\frac{p^{2}}{S_{1}+S_{2}}\right) q_{s}^{2}+\frac{K S_{4}^{*} h^{2} q_{s}^{2}}{\gamma}\right] / \Delta_{0}, \\
\Delta_{0}= & \frac{K h^{2}}{\gamma}\left\{q_{s}^{2}-\left(\xi^{2}+\frac{p^{2}}{S_{1}+S_{2}}\right)\right\}, s=1,2,3, \\
B_{1}= & B_{4}=F_{0}\left(a_{3}-a_{2}\right) / 2 q_{1} \Delta_{2}, B_{2}=B_{5}=F_{0}\left(a_{1}-a_{3}\right) / 2 q_{2} \Delta_{2}, \\
B_{3}= & B_{6}=F_{0}\left(a_{2}-a_{1}\right) / 2 q_{3} \Delta_{2} \text { and } \Delta_{2}=S_{13}\left\{\left(a_{2} b_{3}-a_{3} b_{2}\right)\right. \\
& \left.+\left(a_{3} b_{1}-a_{1} b_{3}\right)+\left(a_{1} b_{2}-a_{2} b_{1}\right)\right\} .
\end{aligned}
\end{aligned}
$$

\section{Inversion of the transformation}

The transformed displacements and stresses are functions of $z$, the parameters of Laplace and Fourier transforms $p$ and $\xi$ respectively, and hence are of the form $\tilde{f}(\xi, z, p)$. To get the function $f(x, z, t)$ in the physical domain, first we invert the Fourier transform using,

$$
\begin{aligned}
\tilde{f}(x, z, p) & =\frac{1}{2 \pi}-\int_{-\infty}^{\infty} \exp (i \xi x) \tilde{f}(\xi, z, p) \mathrm{d} \xi \\
& =\frac{1}{\pi} \int_{0}^{\infty}\left\{\cos (\xi x) \tilde{f}_{e}-i \sin (\xi x) \tilde{f}_{o}\right\} \mathrm{d} \xi
\end{aligned}
$$

where $\tilde{f}_{e}$ and $\tilde{f}_{o}$ are even and the odd parts of the functions $\tilde{f}(\xi, z, p)$ respectively. Thus expression (81) gives the Laplace transform $\tilde{f}(x, z, p)$ of the function $f(x, z, t)$.

Now, for fixed values of $\xi, x$ and $z$, the function $\tilde{f}(x, z, p)$ in the expression (81) can be considered as the Laplace transform $\tilde{g}(p)$ of some function $g(t)$. Following Honig \& Hirdes (1984), the Laplace transformed function $\tilde{g}(p)$ can be inverted as given below:

The function $g(t)$ can be obtained by using

$$
g(t)=(1 / 2 \pi i) \int_{C-i \infty}^{C+i \infty} \exp (p t) \tilde{g}(p) \mathrm{d} p
$$

where $C$ is an arbitrary real number greater than all the real parts of the singularities of $g(p)$. Taking $p=C+i y$, we get

$$
g(t)=[\exp (C t) / 2 \pi] \int_{-\infty}^{\infty} \exp (i t y) \tilde{g}(C+i y) \mathrm{d} y .
$$

Now, taking $\exp (-C t) g(t)$ as $h(t)$ and expanding it as Fourier series in $[0,2 L]$, we obtain approximately the formula

$$
g(t)=g_{\infty}(t)+E_{D},
$$


where

$$
\begin{aligned}
g_{\infty}(t) & =\left(C_{0} / 2\right)+\sum_{k=1}^{\infty} C_{k}, 0 \leq t \leq 2 L, \\
C_{k} & =[\exp (C t) / L] \operatorname{Re}\{\exp (i k \pi t / L) \tilde{g}(C+i k \pi / L)\} .
\end{aligned}
$$

$E_{D}$ is the discretization error and can be made arbitrarily small by choosing large enough $C$.

Since the infinite series in (85) can be summed up only to a finite number of $N$ terms, so the approximate value of $g(t)$ becomes

$$
g_{N}(t)=\left(C_{0} / 2\right)+\sum_{k=1}^{N} C_{k}, 0 \leq t \leq 2 L,
$$

Now, we introduce a truncation error $E_{T}$ that must be added to the discretization error to produce the total approximation error in evaluating $g(t)$ using the above formula. The discretization error is reduced by using the 'Korrecktur method' and the ' $\varepsilon$-algorithm' is used to reduce the truncation error and hence to accelerate the convergence.

The Korrecktur method formula, to evaluate the function $g(t)$ is

$$
g(t)=g_{\infty}(t)-\exp (-2 C L) g_{\infty}(2 L+t)+E_{D}^{\prime}
$$

where $\left|E_{D}^{\prime}\right| \ll\left|E_{D}\right|$.

Thus, the approximate value of $g(t)$ becomes

$$
g_{N_{k}}(t)=g_{N}(t)-\exp (-2 C L) g_{N^{\prime}}(2 L+t),
$$

where $N^{\prime}$ is an integer such that $N^{\prime}<N$.

We shall now describe the ' $\varepsilon$-algorithm' which is used to accelerate the convergence of the series in (86). Let $N$ be a natural number and $S_{m}=\sum_{k=1}^{m} C_{k}$ be the sequence of partial sums of (86). We define ' $\varepsilon$-sequence by

$$
\varepsilon_{0, m}=0, \varepsilon_{1, m}=S_{m}, \varepsilon_{n+1, m}=\varepsilon_{n-1, m+1}+\frac{1}{\varepsilon_{n, m+1}-\varepsilon_{n, m}} ; n, m=1,2,3, \ldots
$$

The sequence $\varepsilon_{1,1,} \varepsilon_{3,1}, \ldots, \varepsilon_{N, 1}$ converges to $g(t)-E_{D}-\left(C_{o} / 2\right)$ faster than the sequence of partial sums $S_{m}, m=1,2,3 \ldots$ The actual procedure to invert the Laplace transform consists of (87) together with the $\varepsilon$-algorithm. The values of $C$ and $L$ are chosen according to the criteria outlined by Honig \& Hirdes (1984).

The last step is to calculate the integral in (81). This method for evaluating this integral is described by Press et al (1986), which involves the use of Romberg's integration adaptive step size. This also uses the results from successive refinements of the extended trapezoidal rule followed by extrapolation of the results to the limit when the step size tends to zero.

\section{Numerical results and discussion}

We take the case of aluminium-epoxy composite material (microstretch elastic solid) for numerical calculation. The physical constants used by us are

$$
\begin{aligned}
\rho & =2.19 \mathrm{gm} / \mathrm{cm}^{3}, \lambda=7.59 \times 10^{10} \text { dynes } / \mathrm{cm}^{2}, \mu=1.89 \times 10^{10} \text { dynes } / \mathrm{cm}^{2}, \\
K & =0.0149 \times 10^{10} \text { dynes } / \mathrm{cm}^{2}, \gamma=0.0268 \times 10^{10} \text { dynes }, j=0.00196 \mathrm{~cm}^{2}, \\
\lambda_{o} & =0.037 \times 10^{10} \text { dynes } / \mathrm{cm}^{2}, \lambda_{1}=0.037 \times 10^{10} \text { dynes } / \mathrm{cm}^{2}, \\
\alpha_{o} & =0.61 \times 10^{10} \text { dynes. }
\end{aligned}
$$


We have considered $\varepsilon=K / \mu$ (dimensionless) as coupling coefficient. The computations were carried out for the three different values of time namely $t=0.025 \mathrm{~s}, 0.075 \mathrm{~s}, 0.125 \mathrm{~s}$ for fixed $\varepsilon=0.0078$ and for three values of coupling coefficient namely $\varepsilon=0.0078,0 \cdot 01,0 \cdot 125$ for fixed time $t=0.075 \mathrm{~s}$, at $z=1$ and $h=1 \mathrm{~cm}$ in the range $0 \leq x \leq 10$. The numerical values for normal displacement, couple stress and normal force stress in case of microstretch elastic medium are large as compared to micropolar elastic medium, so to compare the results of both theories and to show their influence in single graph we magnify the values of above three quantities for micropolar elastic medium by multiplying each value by 10 .

Figure 1 shows the variation of normal displacement for micropolar and microstretch elastic solids. For microstretch elastic solid it oscillates in the range $0 \leq x \leq 10$ and for micropolar elastic solid it decreases in the range $0 \leq x \leq 4,9<x \leq 10$ and increases in the range $4<x \leq 9$ as time increases from 0.025 s to 0.125 s for fixed value of $\varepsilon=0.0078$. Figure 2 shows the variation of normal displacement for both theories which oscillates in the microstretch elastic medium in range $0 \leq x \leq 10$, whereas in micropolar elastic solid it decreases in range $0 \leq x \leq 4,9<x \leq 10$ and increases in the range $4<x \leq 9$ as $\varepsilon$ increases from 0.0078 to 0.0125 for fixed value of time $t=0.075 \mathrm{~s}$.

Figure 3 shows the variation of couple stress for both media. For microstretch elastic medium it oscillates in the range $0 \leq x \leq 10$ whereas for micropolar elastic medium it increases in the range $0 \leq x \leq 3.5$ and decreases in the range $3.5 \leq x \leq 10$ as time increases from $0.025 \mathrm{~s}$ to $0.125 \mathrm{~s}$ for fixed value of $\varepsilon=0.0078$. Figure 4 shows the variation of couple stress for both media which oscillates in microstretch elastic medium in range $0 \leq x \leq 10$ whereas in micropolar elastic medium it increases in the range $0 \leq x \leq 3.5$ and decreases in the range $3.5<x<10$ as $\varepsilon$ increases from 0.0078 to 0.0125 for fixed value of time $t=0.075 \mathrm{~s}$.

The variation of normal force stress for micropolar and microstretch elastic solids have been shown in figure 5 which oscillates in the range $0 \leq x \leq 10$ as time increases from $0.025 \mathrm{~s}$ to $0.125 \mathrm{~s}$ for fixed value of $\varepsilon=0 \cdot 0078$. Figure 6 shows the normal force stress for both media which oscillate in the range $0 \leq x \leq 10$ as $\varepsilon$ increases from 0.0078 to 0.025 for a fixed value of time $t=0.75 \mathrm{~s}$.

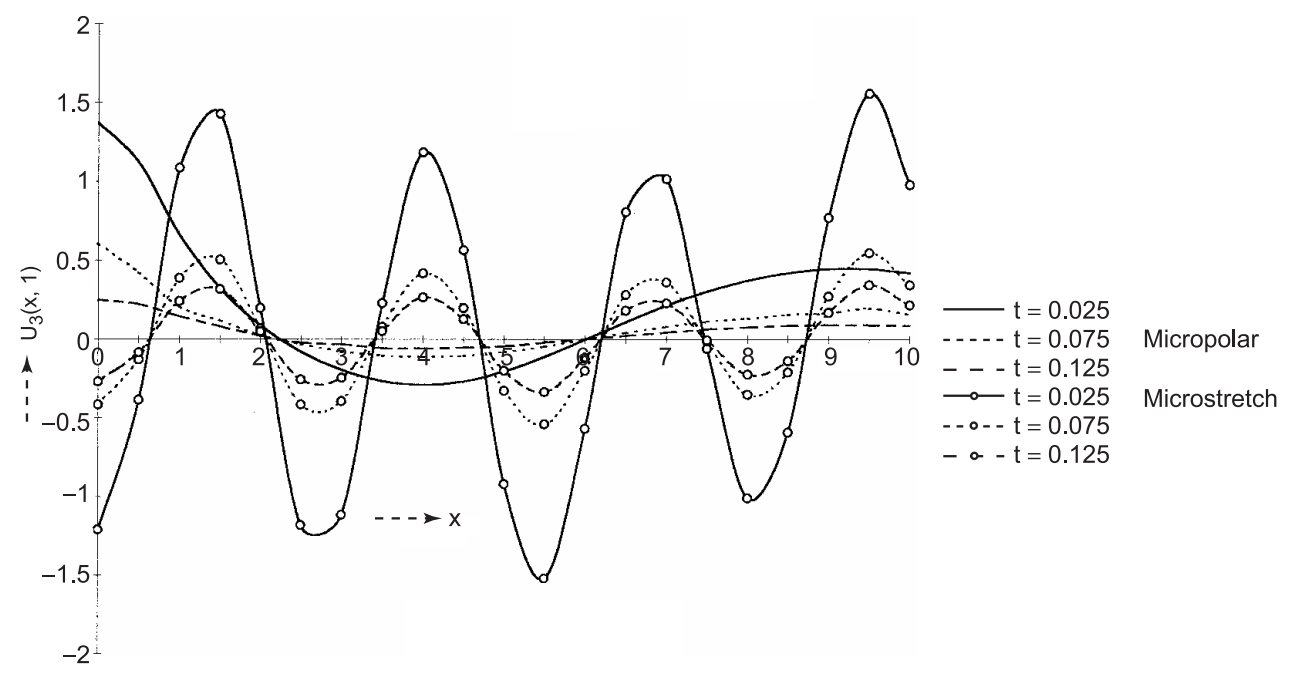

Figure 1. Normal displacement $U_{3}(x, 1), U_{3}=\left(2 / F_{0}\right) u_{3}$. 


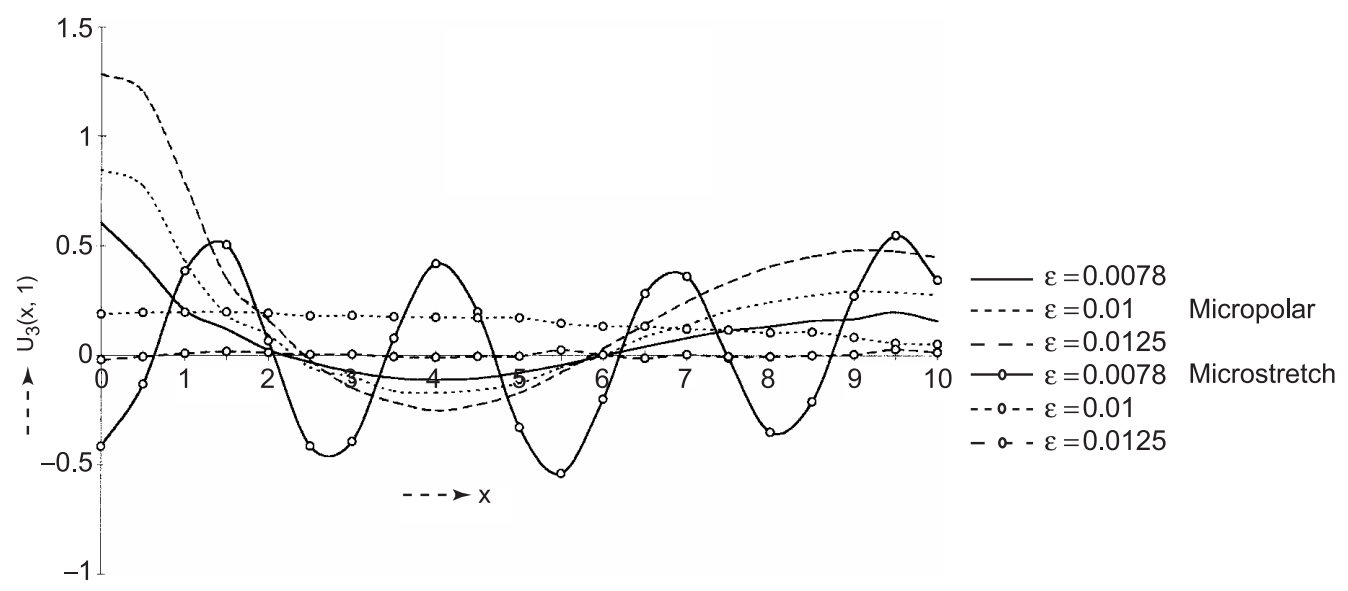

Figure 2. Normal displacement $U_{3}(x, 1), U_{3}=\left(2 / F_{0}\right) u_{3}$.

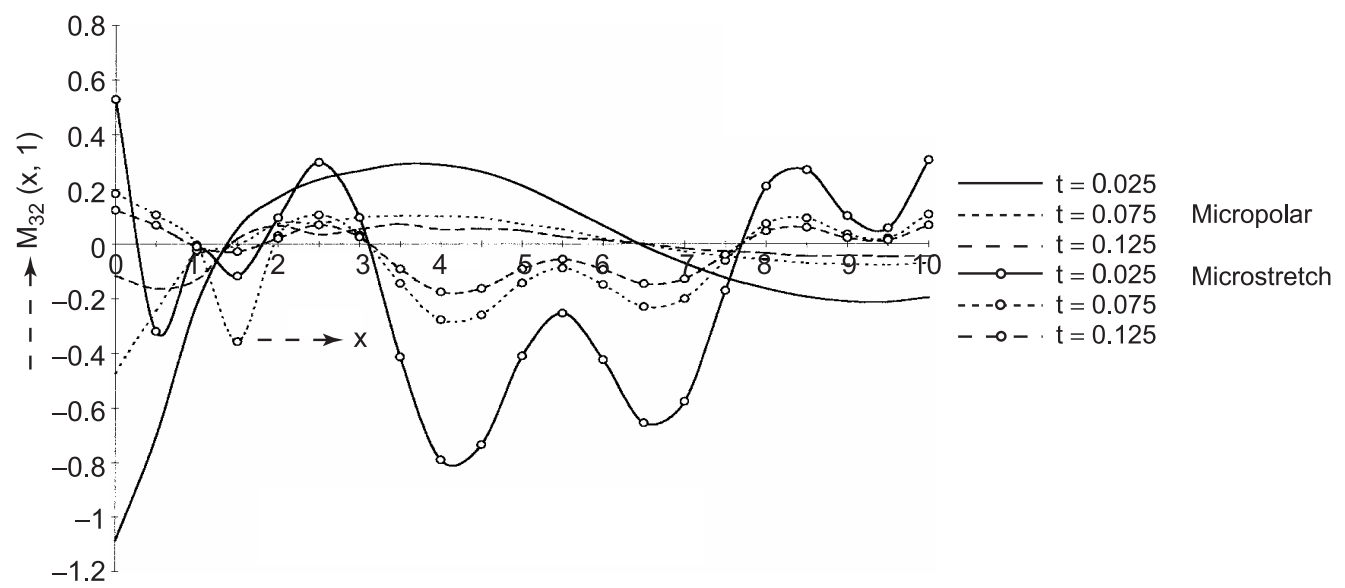

Figure 3. Couple stress $M_{32}(x, 1), M_{32}=\left(2 / F_{0}\right) m_{32}$.

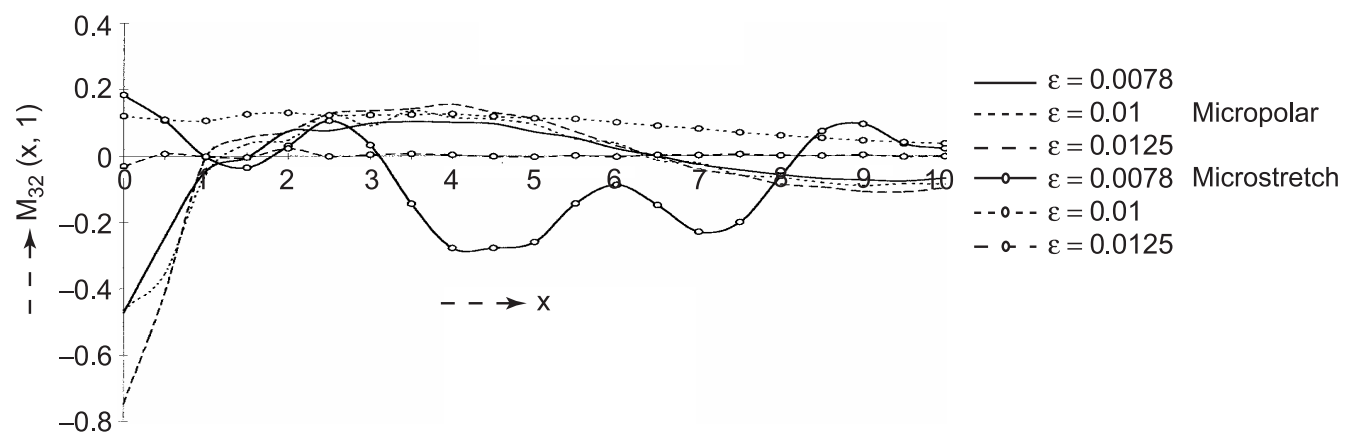

Figure 4. Couple stress $M_{32}(x, 1), M_{32}=\left(2 / F_{0}\right) m_{32}$. 


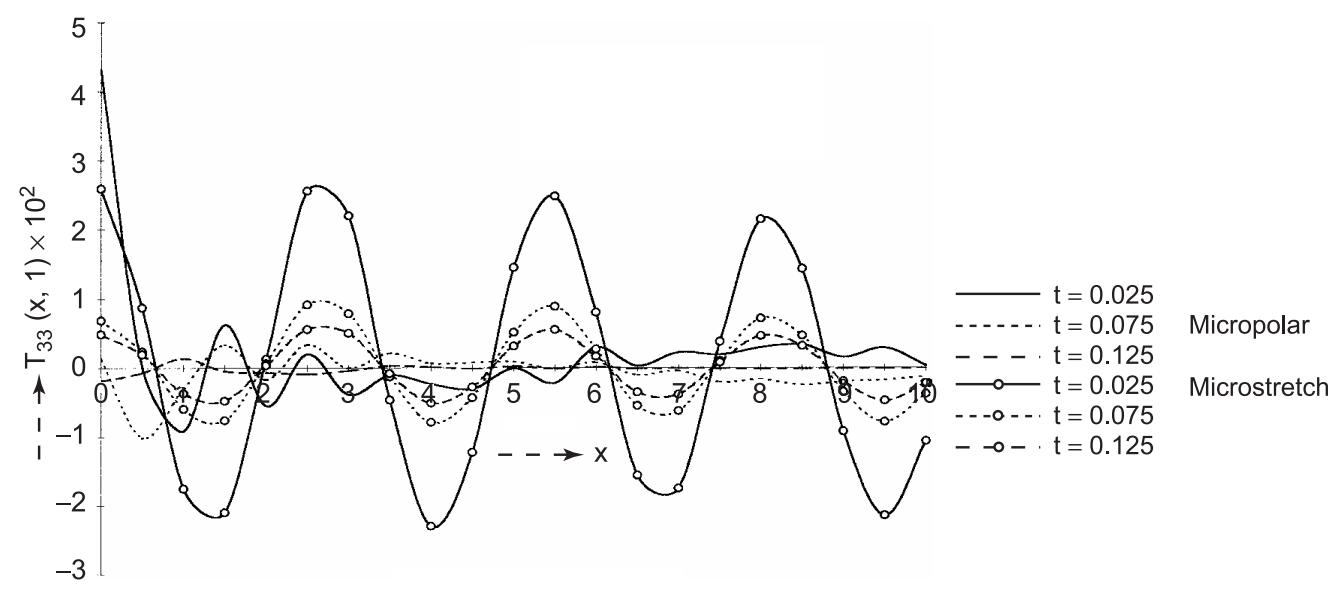

Figure 5. Normal force stress $T_{33}(x, 1), T_{33}=\left(2 / F_{0}\right) t_{33}$.

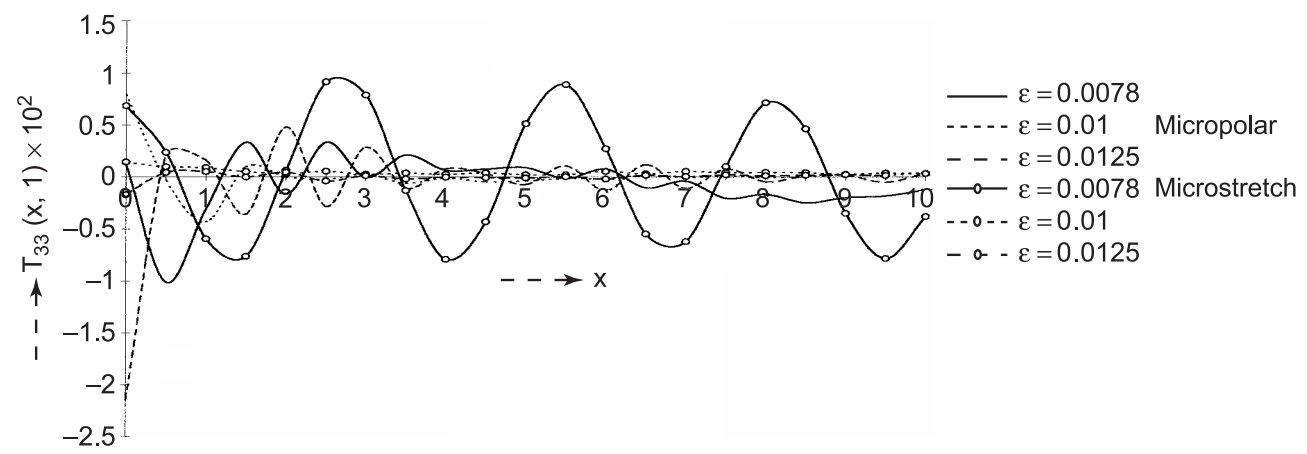

Figure 6. Normal force stress $T_{33}(x, 1), T_{33}=\left(2 / F_{0}\right) t_{33}$.

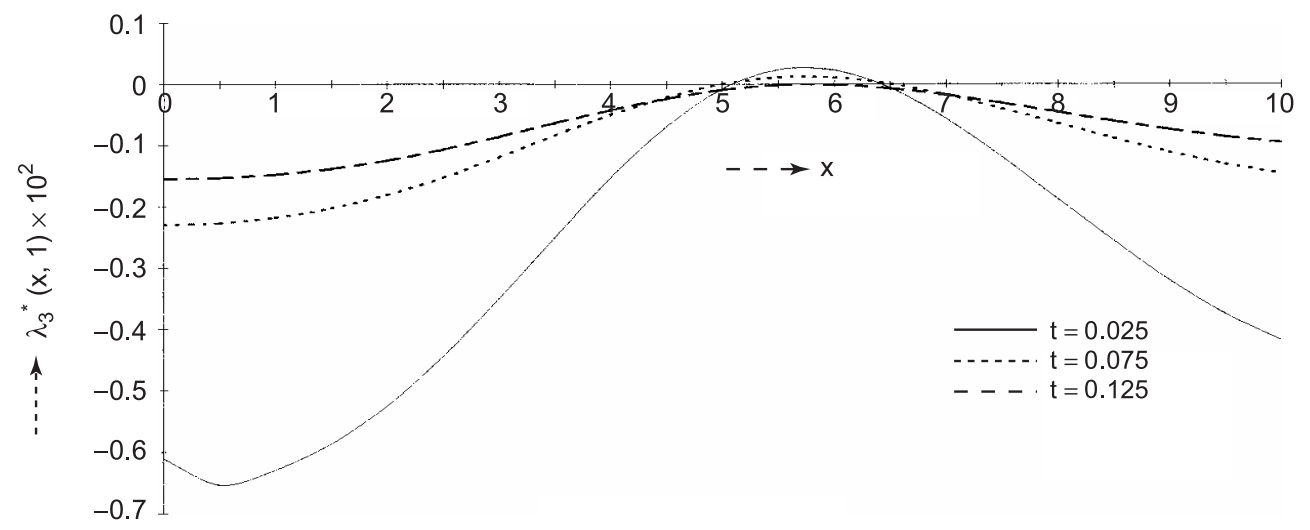

Figure 7. First moment $\lambda_{3}^{*}(x, 1), \lambda_{3}^{*}=\left(2 / F_{0}\right) \lambda_{3}$. 


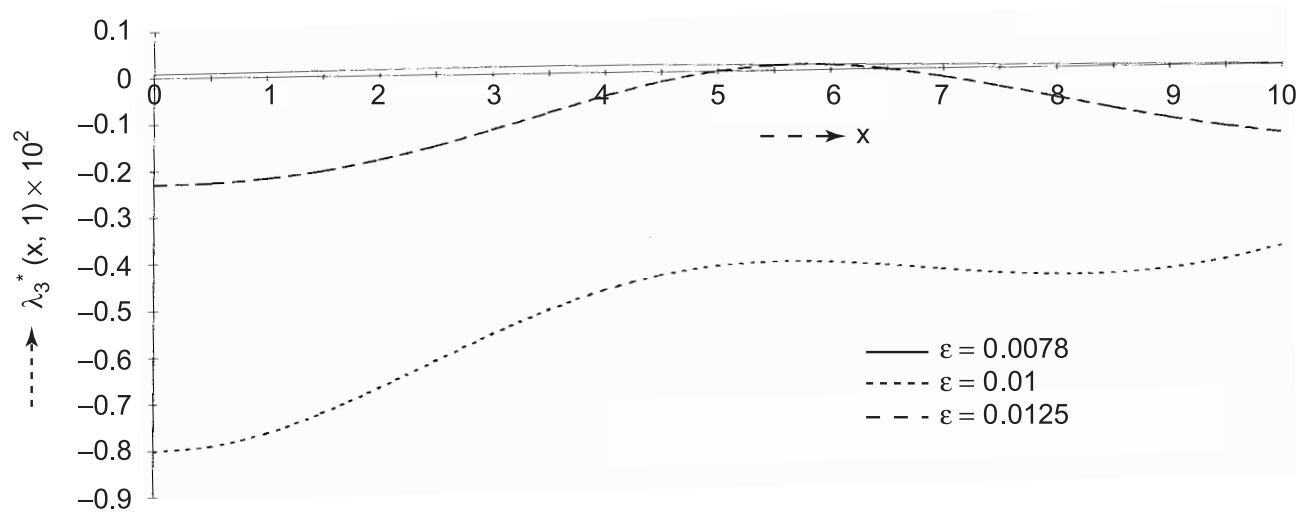

Figure 8. First moment $\lambda_{3}^{*}(x, 1), \lambda_{3}^{*}=\left(2 / F_{0}\right) \lambda_{3}$.

First moment for microstretch elastic medium increases in the range $0 \leq x \leq 5.5$ and decreases in the range $5 \cdot 5<x \leq 10$ as time increases from 0.025 s to $0 \cdot 125$ s for fixed value of $\varepsilon=0.0078$ as shown in figure 7. First moment for microstretch elastic medium increases in the range $0 \leq x \leq 5.5$ decreases in the range $5.5<x \leq 10$ as $\varepsilon$ increases from 0.0078 to 0.025 for fixed time $t=0.075 \mathrm{~s}$ as shown in figure 8 .

\section{Conclusion}

Normal displacement, couple stress and normal force stress for microstretch elastic solids are more as compared to micropolar elastic solids. It is also observed from the graphs of normal displacement, couple stress, normal force stress and first moment for microstretch elastic medium that they are sinusoidal. Thus, stretch plays a significant role in the mechanics of micropolar materials.

\section{References}

Bofilt F, Quintanilla R 1995 Some qualitative results for the linear theory of thermo-microstretch elastic solids. Int. J. Eng. Sci. 33: 2115-2125

Das N C, Dass S N, Das B 1983 Eigenvalue approach to thermoelasticity. J. Thermal Stresses 6: 35-43

Eringen A C 1966 Linear theory of micropolar elasticity. J. Math. Mech. 15: 909-924

Eringen A C 1971 Micropolar elastic solids with stretch. Ari Kitabevi Matbassi 24: 1-18

Eringen A C 1990 Theory of thermo-microstretch elastic solids. Int. J. Eng. Sci. 28: 1291-1301

Honig G, Hirdes U 1984 A method for the numerical inversion of the Laplace transform. J. Comput. Appl. Math. 10: 113-132

Iesan D, Nappa L 1994 Saint-Venant's problem for microstretch elastic solids. Int. J. Eng. Sci. 32: 229-236

Iesan D, Quintanilla R 1994 Existence and continuous dependence results in the theory of microstretch elastic bodies. Int. J. Eng. Sci. 32: 991-1001

Iesan D, Pompei A 1995 On the equilibrium theory of the microstretch elastic solids. Int. J. Eng. Sci. 33: $399-410$

Iesan D, Nappa L 1995 Extension and Bending of microstretch elastic circular cylinders. Int. J. Eng. Sci. 33: 1139-1151 
Kumar R, Gogna M L 1992 Steady-state response to moving loads in micropolar elastic medium with stretch. Int. J. Eng. Sci. 30: 811-820

Kumar R, Singh B 1996 Wave propagation in a micropolar generalized thermoelastic body with stretch. Proc. Indian Acad. Sci (Math.Sci). 106: 183-199

Mahalanabis R K, Manna J 1989 Eigenvalue approach to linear micropolar elasticity. Indian J. Pure Appl. Math. 20: 1237-1250

Press W H, Teukolsky S A, Vellerling W T, Flannery B P 1986 Numerical reciples in FORTRAN (Cambridge: University Press)

Saxena H S, Dhaliwal R S 1990 On the axisymmetric and plane strain problems of coupled thermoelasticity. J. Thermal Stresses 13: 161-175

Sharma J N, Chand D 1992 On the axisymmetric and plane strain problems of generalized thermoelasticity. Int. J. Eng. Sci. 30: 223-230

Sharma J N, Kumar V 1996 On the axisymmetric problems of generalized anisotropic thermoelasticity. J. Thermal stresses. 19: 781-794

Singh B, Kumar R 1998 Wave propagation in a generalized thermo-microstretch elastic solid. Int. J. Eng. Sci. 36: 891-912 